\title{
La innovación y las grandes empresas*
}

\author{
Juan Urrutia Elejalde \\ Catedrático de Fundamentos \\ del Análisis Económico
}

\begin{abstract}
RESUMEN
En este trabajo he tratado de aproximarme al S.C.T .español dentro del contexto privatizador, incluso cuando se trate de una empresa de producción de ciencia básica, con una referencia específica a la propiedad intelectual y en un horizonte que contempla la R.S.C. La primera constatación es que este sistema apenas ha cambiado nada en los últimos diez años y exhibe los mismos cuellos de botella que se localizan precisamente en cuestiones de propiedad. No es de extrañar por lo tanto que el diagnóstico sobre la aportación de las grandes empresas no sea muy halagüeño. Creo que no es exagerado afirmar que no han sabido aprovechar las posibilidades que les ofrece su poder de mercado, posiblemente porque no han tenido necesidad de hacerlo. Pero esta falta de experiencia hace dudosa su inmediata aportación a la modificación del sistema.

* Este trabajo tiene su origen en un encargo específico de examinar la relación entre grandes empresas e innovación que me hizo la Real Académica de Ciencias Morales y Políticas a través de J.A. Sánchez Asiaín. El examen exigía la conversación en profundidad con responsables de grandes empresas de diversos sectores y para acceder a ellos conté con la ayuda inestimable de COTEC y especialmente de Juan Mulet. Además de a José Ángel y a Juan debo agradecer la ayuda prestada por los señores Benjumea, Fernández Sousa y Lada, quienes me concedieron pacientemente parte de su valioso tiempo y, en el caso de Lada, además, unos comentarios que agradezco. Ninguno de los citados es responsable de mis opiniones.
\end{abstract}


La innovación es hoy crucial. En un mundo globalizado, en efecto, la división internacional del trabajo no es la consecuencia de costes salariales relativos; sino más bien de la calidad diferencial del producto y/o del diferencial en productividad. En la sociedad del conocimiento esos diferenciales radican en la cantidad de ciencia y tecnología aplicada en su creación, es decir en la cantidad de inteligencia incorporada. Y la reducción en el coste de la incorporación de esa inteligencia depende de la utilización de las tecnologías de la información y la comunicación (TIC) que, a su vez, son objeto de una innovación continua.

Hablar hoy de innovación es hablar de una cadena de ciencia, tecnología y mercado que hace de esa innovación algo que va más allá del progreso tecnológico (del que la ciencia económica se ha hecho eco desde hace tiempo) y que ha adquirido independencia conceptual y tratamiento sistemático a partir del trabajo de Paul Romer relativo al crecimiento endógeno. Este concepto y este tratamiento recientes se reflejan en la consideración específica de un sistema de ciencia y tecnología (S.C.T.), en parte público y en parte privado, a través del cual la ciencia y la tecnología primero se crean y luego transitan hacia el mercado. Un sistema en el que las empresas privadas juegan un papel crucial para lograr el aumento de productividad que se persigue.

El discurso sobre innovación es ya muy extenso, muy rico en ideas y abundante en referencias empíricas. La singularidad del presente tratamiento es que la innovación es contemplada en el contexto de la privatización de la ciencia, o de la empresa de base científica, con referencia específica a la propiedad intelectual y en el horizonte de la responsabilidad social corporativa (R.S.C.). Este alejamiento relativo de tratamientos más convencionales está justificado por su capacidad para acomodar de una manera muy natural el análisis de la aportación de las grandes empresas a través de la opinión de altos responsables de algunas de ellas.

Estas opiniones aparecerán claramente subrayadas a lo largo de todo el texto que, comenzando con unos comentarios introductorios, presta luego atención a los S.C.T. enfatizando la posibilidad de una ciencia privatizada que, en buena parte, podría ser hecha por unas empresas, relativamente grandes, que tienen capacidad tractora y/o de absorción y en cuya aportación a la innovación puede, finalmente, jugar un papel importante la propiedad intelectual y la R.S.C. 


\section{INTRODUCCIÓN}

La noción de innovación, con los perfiles que hoy presenta, es el resultado de una historia no muy dilatada en el tiempo que no es difícil de describir en sus aspectos tanto macro como microeconómicos.

Es especialmente fácil de entender en sus aspectos macroeconómicos pues es resultado de una historia bastante lineal. En los años 50 del pasado siglo la idea de competencia perfecta, caracterizada por la existencia de un gran número de agentes, no se había normalizado del todo como propia de una visión estática del sistema de mercado y se hablaba, más bien, de un sistema capitalista que, caracterizado por la libre entrada de nuevas empresas era el motor del crecimiento. El sistema capitalista sería la gran máquina creativa y cabía la defensa, impensable hoy, del monopolio como generador de progreso tecnológico, el aspecto productivo de la innovación ${ }^{1}$. En los años 60 y 70 esta idea de progreso tecnológico se incorpora en los modelos de crecimiento más o menos agregados que no eran propiamente dinámicos sino la traducción dinámica del modelo neoclásico de Equilibrio General ${ }^{2}$. En este contexto las preguntas eran si este progreso tecnológico era ahorrador de trabajo o de capital o si era incorporado o desincorporado y el énfasis basculaba sobre su capacidad de replicar la contabilidad del crecimiento. No es hasta la década de los 80 que esas ideas se traducen de tal manera que se empieza a considerar, en el contexto de las teorías de crecimiento endógeno, tanto a la I+D como al capital humano, como los factores que determinan lo que se denomina el residuo de Solow o la productividad total de los factores (P.T.F.). Es gracias a estas ideas que se comienza a prestar atención a la vieja institución de la Ciencia Abierta $^{3}$ como base por esa innovación que acaba incrementando la productividad y mejorando el nivel de vida de los ciudadanos del país que se atreve a tomársela en serio.

A partir de este punto la indagación sobre el origen del incremento de la productividad desplaza su centro neurálgico desde el aparato productivo hacia las institu-

1 Véase Baumol (2002). La importancia del monopolio aparece ya en Schumpeter (1942) y se destaca también en Baumol (1951).

2 Véase Solow (1956).

${ }^{3}$ Cfr. David (1998). 
ciones sociales que fomentan o dificultan la adopción de la innovación que la ciencia y la tecnología hacen posibles. Manteniéndome dentro de un marco más bien macroeconómico, reitero lo que he argüido a menudo, la importancia crucial de la cultura científica y tecnológica distinguiendo entre las dos actitudes que corresponden a la conocida metáfora de Atenas y Manchester que Freeman Dysson puso en circulación. Atenas sería como la patria de los científicos que están a la búsqueda de un principio unificador que lo explique todo, mientras que Manchester alberga a aquellos otros científicos que disfruta con la proliferación de artefactos y con el examen minucioso de hechos y objetos. Entre estas dos culturas, sería justamente la Manchesteriana la que estaría en el origen de la innovación, lo que justificará, más adelante, que mi énfasis se deslice hacia la empresa privada.

Este énfasis en la empresa privada nos lleva al último comentario introductorio, de corte microeconómico esta vez. El objetivo de este pequeño estudio es entender la conexión entre Innovación y gran empresa sobre la que ya se puede avanzar algo. Ayer, si recordamos a Schumpeter o Baumol, nos encontrábamos con que, como ya he dicho, el monopolio parecía adecuado para la innovación debido a que la investigación subyacente podría ser financiada más fácilmente por los beneficios extraordinarios propios de este régimen de empresa. Hoy sabemos que innovación y competencia van de la mano. La competencia es propiciada por la innovación y ésta justifica el nacimiento de empresas que destruyen los monopolios previos, quizá surgidos de la mismísima legislación sobre patentes.

Pero sabemos más cosas aunque sean fragmentarias. Tenemos, en primer lugar, esa especie de tópico, que se va haciendo real, de que las TIC traen consigo una especie de aplanamiento de la organización jerárquica lo que reduce los costes de coordinación y hace más posible la adopción de innovaciones en la medida en que éstas tienen que salvar menos obstáculos. Este es un punto muy simple pero muy importante que desgraciadamente no se ve reflejado tal cual en las empresas españolas de un cierto tamaño que yo he podido investigar. A pesar de ello hay que mencionar que algo así ocurre en empresas que están totalmente enraizadas en la investigación y que en otras, muy volcadas en I+D por razones de posicionamiento, la innovación guía las sinergias entre negocios. Pero es que, además y en segundo lugar, la innovación exige creatividad y ésta está especialmente apoyada en lo que he llamado promiscui$\operatorname{dad}^{4}$ y que tiene al menos tres aspectos importantísimos. Debe prestarse atención a la

${ }^{4}$ Véase los dos artículos de J. Urrutia en Expansión en 2005 en donde se ofrecen las referencias pertinentes. Pueden leerse en juan.urrutiaelejalde.org 
promiscuidad intergeneracional de forma que en las empresas compartan ideas y soluciones las generaciones jóvenes, mejor formadas científicamente, con las «viejas», más experimentadas en la solución de problemas prácticos. También importa la promiscuidad social entendiendo por tal la posibilidad de que el talento y los científicos puedan llegar a la cúspide de las decisiones empresariales en un mundo en el que la globalización y la correspondiente deslocalización hacen del talento, y no de lo financiero, el factor escaso. Hasta donde yo he podido saber esta promiscuidad social está lejos de darse conscientemente en las empresas que me han abierto sus puertas aunque, en las que corresponden a ciertos sectores, los técnicos están más involucrados en la toma de decisiones. Estas empresas tampoco parece que estén poniendo en práctica o viéndose obligadas a aceptar, la diversidad cultural que está positivamente correlacionada con la productividad debido a que permite el contraste entre diversas maneras de solucionar problemas prácticos, un tipo de diversidad que, en embargo, las grandes empresas española podrían explorar para sus inversiones fuera de España.

Pues bien, la importancia de la I+D para la P.T.F., la relevancia de la cultura manchesteriana, y las novedades que se empiezan a descubrir en términos microeconómicos nos llevan a replantearnos ese S.C.T. del que tanto se habla.

\section{Sistemas de CiEnCIA Y teCnOlogía}

Hasta muy poco tiempo, tanto la ciencia como la tecnología eran la muestra de un tipo de bien, la invención, que posee unas características tales que hacen de él un bien difícil de proveer óptimamente por el mercado. En primer lugar, el proceso que va desde la invención científica a la innovación tecnológica y su difusión en las industrias hasta llegar al mercado incorporada en bienes concretos, es de resultados muy inciertos, por lo que, en general, no habría nadie dispuesto a financiar el origen del proceso, es decir la investigación básica. De ahí que ésta sólo se lleve a cabo en empresas grandes muy diversificadas, lo que no es el caso en las empresas con las que he entrado en contacto, quizá no tanto por falta de diversificación, sino por falta de tamaño, o en las diversas instituciones investigadoras del sector público. En segundo lugar, la inapropiabilidad esencial del conocimiento parecería exigir que se estableciera un compromiso entre los incentivos a inventar, protegiendo el derecho del inventor mediante patentes que otorgan un monopolio temporal y los beneficios sociales de la difusión de los inventos, que sólo se puede lograr limitando la duración de las patentes y garantizando la existencia de un mercado de royalties y licencias que 
palie las nocivas consecuencias del monopolio. Debido a estas dos razones todavía existe en todos los países una connivencia institucional entre el sector privado y el público que llamamos S.C.T.. En este apartado pretendo focalizar la atención del lector en tres aspectos: la discutible necesidad del carácter público de la empresa científica, la situación española en términos de esfuerzo público y la nueva manera de mirar a las patentes y a la propiedad intelectual en general.

Comenzando por el aspecto descriptivo del S.C.T. español, con relación a otros países, podemos resumirlo en aspectos básicos y cuantificables. En cuanto al imput de un S.C.T. tenemos que mirar al gasto en I+D y a su distribución por financiación y por ejecución. En cuanto al gasto total estamos por debajo de lo que nos corresponde (apenas por encima de un $1 \%$ del PIB), en cuanto a financiación estamos empeñados en pasar del $(2 / 3,1 / 3)$ a favor de lo público a justo lo contrario, $(1 / 3,2 / 3)$, a favor de las empresas privadas; y en cuanto a ejecución sigue siendo cierto que son las empresas privadas las que llevan a cabo el trabajo a partir de las transferencias recibidas del sector público. En cuanto al output de nuestro S.C.T. puede ser muy brevemente resumido. Los resultados puramente científicos convergen con el entorno, aunque menos rápidamente que el PIB. Los resultados tecnológicos, medidos en términos de patentes mejoran; pero no convergen a pesar del reciente esfuerzo de los propios centros públicos de investigación. En consecuencia en términos comerciales nuestro S.C.T. es defectuoso y crecientemente deficitario en relación al entorno con tasas de cobertura de la importación bajas relativamente. En estas circunstancias a nadie puede extrañar que nuestra posición innovadora sea decepcionante ${ }^{5}$.

Esto ha llevado a la formulación del Programa INGENIO que ordena los esfuerzos y programas existentes y se fija unos objetivos claros; pero no rompe con lo que son cuellos de botella importantes. Lo que llamo cuellos de botella tienen que ver con la propiedad, bien de la empresa científica, bien de las invenciones. Trataré ahora de resumir brevemente dos ideas que me parecen interesantes. La primera, que no hay incompatibilidad alguna entre propiedad privada y provisión de ciencia y, la segunda, que la propiedad intelectual en general (y las patentes en particular) pueden ser un freno a la innovación.

${ }^{5}$ La situación no ha cambiado sustancialmente desde la publicación de Lafuente y Oro (1992). Ver también mi comentario a su trabajo aparecido al año siguiente. En este comentario se subrayaba el resultado de Lafuente y Oro relativo al cuello de botella que las empresas representaban y se hacía hincapié en la distinción entre Manchester y Atenas tan brillantemente introducida por Dysson. 
Comencemos por enfocar el asunto de la propiedad privada como un problema de incentivos en un contexto de contratos incompletos. Una empresa es un haz de contratos incompletos en el sentido de que no contemplan todas las contingencias posibles. Concentremos nuestra atención en el caso de una empresa científica y digamos que el contrato central para la formación de esa empresa se firma entre el Estado y una clase especial de agente privado, que denominaré el Científico, y consiste en especificar ex ante las inversiones complementarias de uno y otro. El Estado pone la infraestructura (La ESS, European Spalation Source o fuente de Neutrones por espalación, por ejemplo), que no valora en sí misma más allá del impacto temporal en el PIB y el Científico, que si valora la instalación en sí misma, pone su capital humano, es decir, su capacidad de usar esa instalación. Si este contrato fuera completo estaríamos en presencia de un problema de identificar el óptimo de primer rango, la propiedad sería irrelevante para la eficiencia y no habría problema de incentivos. En un contrato completo, en efecto, no hay problema de incentivos porque el criterio obvio para decidir las respectivas inversiones complementarias es la maximización del beneficio conjunto. Y este es el criterio obvio porque el reparto posterior de este beneficio está especificado perfectamente. Cual sea esta especificación, es decir de quién son los bienes, influye en la distribución; pero no en la eficiencia. Ahora bien parece evidente que un contrato así no puede ser un contrato completo. La ESS citada no es una máquina estándar, sus especificaciones pueden variar a lo largo de su construcción y la aplicación con que el científico ponga en juego su capital humano es también muy variable. Por todo ello habrá muchas circunstancias en las que es imposible verificar si se están cumpliendo los pactos establecidos ex ante. Es claro que cuando este contrato es incompleto, el reparto del beneficio conjunto no puede estar especificado perfectamente en toda contingencia y, en consecuencia, el óptimo de primer rango no es alcanzable. El óptimo de segundo orden dependerá ahora de los incentivos. Pues bien, ahora la propiedad es esencial para los incentivos (y por lo tanto para alcanzar el óptimo de segundo orden) pues determina quién tiene el derecho a continuar con el proyecto en el caso de que, una vez instalada la infraestructura, surja una desavenencia no tenida en cuenta en el contrato entre Estado y Científico.

Pensemos, en segundo lugar, que entre Estado y Científico van a producir ese bien con características de bien público que denominamos ciencia. Supongamos que la propiedad fuera del Estado y que éste hubiera decidido hacer una inversión muy grande. En este caso, si el acuerdo se rompe ante una contingencia no prevista hay dos posibilidades: que el Estado decida interrumpir el proyecto o que decida continuarlo. En la primera eventualidad ha malgastado una gran inversión, en la segunda el rendimiento se lo lleva el Científico, luego cabe pensar que el Estado no tiene 
incentivos a hacer una gran inversión. Supongamos ahora que la propiedad fuera del Científico y que el Estado ha decidido hacer una inversión muy grande. Si el acuerdo se rompiera, al científico le compensa seguir en el proyecto aunque tenga que ceder parte del excedente al Estado porque él, el científico, valora mucho la infraestructura en sí. En consecuencia, el Estado tiene incentivos a hacer una inversión grande. Este es el argumento de Besley y Ghatak $^{6}$ para defender que la propiedad debe asignarse al Científico que aprecia el bien público más que el inversor. Esta conclusión no sólo es coherente con la tendencia detectada, y mencionada en la sección anterior, a que los científicos adquieran poder decisorio en las empresas, sino, lo que es más importante, da juego al llamado capital-riesgo orientándolo de manera sorprendente y rompe unos de los pilares en los que se suele asentar un S.C.T.

El otro pilar de un S.C.T., el de las patentes, también debe ponerse en duda siguiendo algunas ideas recientes. Una de ellas puede resumirse de la siguiente manera: «Si la invención o idea creativa está incorporada en un producto (lo que siempre es el caso), si la reproducción, imitación o copia exigen una cierta formación intelectual o técnica que haga que la imitación nunca sea sin costes ( lo que ocurre en general) y si hay límites a la capacidad de reproducción (lo que es bastante obvio en la mayoría de los casos), el valor descontado presente de las cuasi rentas que recibe el creador inicial en ausencia de copyright o patentes es positivo. Pero es que además, no solamente es positivo, sino que crece a medida que se reducen los costes de reproducción y el producto en el cual la idea se incorpora» ${ }^{7}$. Esto no es fácil de probar formalmente; pero más adelante ofreceré una explicación más sencilla.

\section{El PAPEL DE LAS GRANDES EMPRESAS}

Dentro de los S.C.T. de nuestro entorno siempre se ha pensado que las grandes empresas (definidas no siempre de acuerdo con los mismos parámetros) deberían jugar un papel importante sobre todo porque son las únicas que se pueden permitir una actividad de $\mathrm{I}+\mathrm{D}$ de resultados inciertos porque, precisamente por ser grandes, es

${ }^{6}$ Hart (1976) es el introductor de las ideas principales. El texto se basa en el trabajo de Besley y Ghatak (2001).

7 Este párrafo está extraído de Boldrin y Urrutia (2003), aunque su fundamentación formal se debe a Boldrin y Levine (2002) en donde se especifican condiciones relativas a la elasticidad de la demanda que son necesarias para el resultado que se cita en el texto. 
más probable que sean diversificadas y, en consecuencia, pueden aprovechar mejor los resultados. El cualquier caso, y a pesar de esta idea que parece obvia, la evidencia cuantitativa nos dice que el cuello de botella del S.C.T. está en las empresas cualquiera que sea su tamaño. Y, sin embargo, son las empresas las únicas capaces de innovar realmente de manera que aumente la productividad sin que una mejora sustancial de las cifras de publicaciones o patentes vaya a sustituir su protagonismo. Por eso conviene aclarar que los incentivos fiscales generados que se han ofrecido en el S.C.T. español han sido aprovechados por una miriada de empresas pequeñas; pero apenas han tenido impacto en las grandes quienes las utilizan solo de manera rutinaria. La única explicación posible de esta anomalía es que estas últimas (excepto, naturalmente, aquellas que han llegado a ser grandes justamente por su capacidad innovadora) no han necesitado innovar, al menos hasta ahora, porque gozan de algún tipo de poder de mercado manifestado en ciertas barreras en la entrada a los sectores en los que se mueven. La puesta en marcha del Programa CENIT quizá cambie algo esta actitud un poco pasiva; pero no parece que haya un entusiasmo desmesurado en relación a su puesta en marcha a pesar del éxito inesperado de la primera convocatoria. Todos estos rasgos hacen pensar que se debiera distinguir por sectores.

Dejando aparte esta advertencia ahora debemos explorar el potencial innovador de las grandes empresas en general. Precisamente porque tienen un cierto poder de mercado debemos explorar si hacen uso de la facilidad que les podía proporcionar para innovar. Ese poder de mercado les dota de un cierto poder monopsónico en el mercado de los inputs que utilizan y de un cierto poder monopólico en el mercado de los outputs que producen. El hecho de que la competencia vaya imponiéndose en los sectores en los que muchas empresas grandes operan no debe hacernos olvidar que la noción de empresa tractora está relacionada con el tamaño y con el consiguiente poder de mercado. Si la competencia fuera muy alta en todos los sectores, en ninguno de ellos podríamos hablar de empresas tractoras y tendríamos que limitarnos a estudiar la tracción que el sector público puede ejercer mediante las compras públicas que lleva a cabo de una manera criticada por alguna empresa grande que observa que la dispersión autonómica de las condiciones debilita su capacidad de arrastre.

Pensemos por lo tanto en las exigencias que una empresa grande puede ejercer sobre sus suministradores aguas arriba y en las que puede imponer sobre sus clientes aguas abajo. Vayamos por pasos.

En cuanto a suministradores, distingamos entre la mano de obra y otras empresas que suministran materias primas o productos semielaborados. Pocas veces se piensa que los trabajadores pueden ser agentes innovadores aunque esta posibilidad esta implícita en el movimiento por la calidad dentro del cual existían círculos de calidad 
que pretendían precisamente extraer el conocimiento tácito la mano de obra, más o menos cualificada, para mejorar la calidad y, en consecuencia, la competitividad. Según las conversaciones que he podido tener con altos responsables de empresas grandes, esto ocurre solo en empresas que viven de la innovación; pero no en general, de forma que la organización es tal que no propicia este destape del conocimiento productivo, quizá porque no es el talento el que manda (tal como esperaríamos que ocurriera en un futuro todavía más globalizado de acuerdo con una idea ya apuntada); sino que todavía quién ordena el funcionamiento de una gran empresa es el responsable financiero o el de marketing. Claro que estos responsables procuran reducir el coste financiero y aumentar las ventas respectivamente a veces de forma creativa; pero no es esta innovación la que puede forzar innovaciones productivas en esa empresa ni en ninguna de las suministradoras. Sin embargo sí que es cierto que empresas que quizá sean grandes entre nosotros, pero que son pequeñas en el mercado global en el que están empezando a moverse, muestran una tendencia a organizarse de forma tal que el ingeniero adquiere poder sobre lo financiero o la mercadotecnia. Este movimiento parece dar a entender que quizá las empresas que se mueven en un mercado global, aunque sean pequeñas en ese ámbito, se ven obligadas a dar poder al talento y a reconocer la importancia de la promiscuidad cultural.

Si bien no se piensa a menudo en los trabajadores como fuente de innovación, sí que se piensa en los suministradores que se convertirían en una fuente tal, gracias a las exigencias que una empresa grande puede ejercer sobre ellas. Recordamos que hace unos años el sistema que López Arriortúa implantó en la General Motors, y que quiso trasladar a la Vokswagen, parecía muy prometedor. Reducía el coste para la gran empresa exigiendo que los proveedores se instalarán al lado de las plantas de esa gran empresa de forma que, además de minimizar el coste de transporte, se maximizaba la rapidez de la respuesta a las exigencias técnicas. No me cabe duda de que las empresas grandes de nuestro S.C.T. estudiaron este sistema y siguieron cuidadosamente sus resultados La experiencia no fue imitada porque no prosperó allí donde se implantó. Y su fracaso tuvo un origen que es instructivo. El suministrador se sintió maltratado por la tendencia de la gran empresa demandante a abusar de él y a no proporcionarle un horizonte claro contractualmente definido. La consecuencia fue que el proveedor dejó de valorar las presentes ventajas y, si pudo, acabó buscando un cliente alternativo. Hoy lo que se observa en los sectores más necesitados de innovación, es una especie de «partenariado» entre la gran empresa del sector y sus suministradores.

Este fracaso de la vía más clara para que una empresa grande pueda mejorar la innovación, oculta a veces lo contraproducente que son otras posibilidades que su poder de mercado otorga a las grandes empresas. Muy a menudo esas grandes 
empresas, de acuerdo con sus propios intereses, tratan de «explotar» financieramente su poder exigiendo ventajas financieras. Esta ha sido la acusación constante a las grandes superficies y, en la medida en que la acusación fuera entonces o siga siendo cierta, tendríamos que esas ganancias financieras eliminan o suavizan la presión a innovar por sí mismas o a tratar de forzar esa innovación en sus suministradores.

No parece pues que podamos apoyarnos en las grandes empresas a través de su poder de mercado aguas arriba. Más prometedora es su capacidad de aprender de sus clientes aguas abajo si son capaces de no apretarles demasiado. Los clientes de una gran empresa, por ejemplo de telecomunicaciones, están en posición de exigir de esa gran empresa mejoras tecnológicas que pueden obtener de otra gran empresa que opere en ese sector aunque éste no sea del todo competitivo. Mientras los costes de cambio sean altos y las autoridades de la competencia o las asociaciones de consumidores no alcancen mayor sensibilidad y poder respectivamente, esta fuente de innovación no parece muy prometedora a corto plazo, aunque sea una fuente evidente de innovación a medio plazo.

En mi opinión la única vía de tracción utilizable pasa por el enorme impacto que pueden tener los consumidores especialmente cuando lo son de un sector como la energía, o la telefonía, que cuenta con un enorme número de ellos. Las nuevas tecnologías de la información y de la comunicación informáticas (TIC) permiten una explotación sistemática y profunda de la base de datos de la clientela. Esta explotación es doble a efectos de innovación. Puede, primero, extraer de esa clientela muchas y variadas ideas innovadoras. Esto es algo que este tipo de gran empresa está empezando a hacer solo de manera muy tímida. Pero también puede, en segundo lugar, utilizar esa base de datos para organizar una comunidad identitaria (identificada por su consumo) organizada en red en cuyo seno pueden surgir muchos negocios nuevos. No me refiero a ese efecto-red que surgiría por ejemplo en telefonía, pero no en electricidad. Me refiero a que esa gran empresa con millones de clientes podría organizar algo como un e-Bay interno que le genere nuevos ingresos, fidelice a la clientela y de hecho cree nuevos mercados. ${ }^{8}$. Sin embargo, no he detectado en las empresas a las que me he podido acercar una sensibilidad hacia este punto, posiblemente porque los miembros de esas comunidades lo son de varias a la vez.

\footnotetext{
${ }^{8}$ Esta idea aparece con toda claridad en la correspondiente sección de Urrutia (2003).
} 
La conclusión hasta el momento parece ser que no hay nada en el poder de mercado, más o menos grande, que privilegie a las grandes empresas como tractoras y facilitadoras de la innovación. Pero todavía tenemos que considerar dos extremos importantes. Es necesario pensar en grandes empresas que, aunque operan en suelo nacional, son filiales o sucursales de una empresa multinacional radicada en otro lugar y preguntarnos si podemos esperar absorber innovación a través de ellas. Y también es necesario pensar en la influencia que la regulación puede ejercer en la innovación en un sector regulado como muchos en los que se mueven las grandes empresas.

La existencia no marginal de innovación por parte de las subsidiarias extranjeras en suelo nacional, hace difícil la separación nítida entre S.C.T. nacionales. A pesar de esta dificultad más bien de cuantificación, podemos decir que su influencia será en general buena ya que difundirá las buenas prácticas investigadoras entre sus suministradores y clientes. Su influencia, sin embargo, puede estar subestimada debido a que muchas de las patentes generadas se registran en el país de origen. Y, a su vez, también es posible que la capacidad de absorción del país de acogida sea limitada. Es pues difícil aventurar una diagnosis sobre la conveniencia de la atracción de esas subsidiarias a falta de estudios empíricos de casos concretos ${ }^{9}$.

Otro factor distorsionador de la imagen que nos podemos hacer de la influencia de las grandes empresas en la innovación es el de la regulación. Las grandes empresas trabajan en sectores tradicionalmente regulados, como el farmacéutico, el de comunicaciones o el energético. Cuando se trata de simple fijación de tarifas hay que tener mucho cuidado en que la posible «persuasión» sobre el regulador no sustituya a la necesidad de innovar derivada de una fijación de tarifas menos «política». Pero lo más interesante es cuando se trata de un sector realmente innovador en el que hay que pasar por sucesivas pruebas antes de que el producto sea aprobado para su venta al público. Esto impone un retardo sustancial en la innovación que exige un mayor capital y produce una discontinuidad en la creación de valor. Raramente se tienen en cuenta estos extremos a la hora de diseñar programas públicos de promoción de la investigación.

Y, para terminar deberíamos pensar en las propias barreras a la entrada como condicionantes de la innovación. La teoría y la evidencia parecen sugerir que una mayor amenaza de entrada en un sector - como podría representar la liberalización de sectores con grandes empresas y la consolidación fomentada por la globalización — ace-

${ }^{9}$ Este comentario proviene del proyecto de tesis doctoral de Guimón( 2005).

10 Véase el trabajo de Aghion et al. 
lera la innovación en sectores punteros tecnológicamente mientras que la retarda o inhibe en sectores maduros ${ }^{10}$.

El aumento de la competencia en general elimina el poder de mercado y debilita la confianza en la capacidad de las grandes empresas para empujar la innovación. Sin embargo si trabajan en sectores tecnológicamente avanzados, estas empresas grandes incrementarían su intento de innovar ellas mismas. Esto, junto con la idea de utilizar la base de datos de la clientela en la medida de lo posible, quiere decir que la importancia de la gran empresa a efectos innovadores no está en su poder de mercado y en la posibilidad que tiene de forzar a los suministradores a innovar; sino en el potencial que ellas mismas tienen empujadas, precisamente, por la erosión de su poder de mercado y, recordemos, por las posibilidades, quizá no tan inmediatas, que les otorga su numerosísima clientela.

\section{INNOVACIÓN Y GRANDES EMPRESAS}

La R.S.C. parece algo alejada de la innovación; sin embargo en la medida que son las grandes empresas las que van a tener que dar cuentas de su labor en este campo, su actividad de mecenazgo puede llegar a tener incidencia sobre la innovación de la que son, o pueden ser, protagonistas. La conexión entre R.S.C. e Innovación está en la posible tendencia del mecenazgo asociado a la primera a bascular hacia la ciencia y no hacia la cultura como hasta ahora. Si eso ocurriera el papel del sector público y su gasto podría disminuir de acuerdo con las intenciones del Programa INGENIO y la posibilidad de una privatización de la generación de ciencia, incluso básica, se vería reforzada.

Para hacer comprensibles las relaciones afirmadas en el párrafo anterior, tengo que volver al problema de la propiedad intelectual, tanto mercantil (patentes industriales) como civil (derechos de autor o de copia-copyright). Podría argüir que los creadores — sean científicos o sean artísticos y culturales - podrían no necesitar incentivos para la creación o invención pues suelen trabajar por la reputación; pero no me hace falta porque aunque no hubieran derechos de propiedad intelectual, los creadores crearían siempre que hubiera un intermediario que demandara su creación estableciendo con él una relación como la existente entre Linux y Red Hat. Pero para que un intermediario como Red Hat exista se necesita una demanda final de productos de la creatividad. Pensemos en empresas industriales (de base científica) o en empresas culturales y preguntémonos en qué condiciones demandarán productos innovadores que incorporan creatividad. 
Eric Raymond ${ }^{11}$ señala las siguientes cinco condiciones que yo expongo a mi manera: (i) La confianza en la calidad del producto de que se trate es crítica para la empresa demandante. Esto es cierto para una empresa farmacéutica; pero es dudoso que lo sea para una editorial. (ii) No hay mejor manera de verificar esa calidad que la «revisión por pares», los del autor de la innovación. Mientras en el mundo cultural el «ojo» del crítico o del propio editor es crucial, en el mundo industrial no existen esas figuras o equivalentes por lo que la «revisión por pares« es imprescindible. (iii) La empresa que demanda la innovación no debiera depender de un suministrador único, una condición que no tiene sentido por ejemplo en el teatro The Globe al que le basta Shackespeare. (iv) El lenguaje de la innovación conforma la comunicación interna de la empresa, algo que no ocurre por ejemplo en una casa editorial cuyos directivos pueden ignorar el lenguaje periodístico, digamos. (v) La necesidad de tener ese tipo de comunicación interna homogeneizada alrededor de la innovación es «conocimiento público», algo que resulta poco creíble en el mundo cultural.

Pues bien, si se dan estas condiciones, nos encontramos con que, dada la idiosincrasia de los creadores — científicos o culturales - y la existencia - real o potencial — de intermediarios, habrá un mercado para esas innovaciones sin necesidad de que estén protegidas por la propiedad intelectual.

Antes de sacar las conclusiones pertinentes relativas a la R.S.C., quiero sembrar ahora la duda sobre la importancia que, con mucho atraso, se le está dando hoy en buena parte de nuestro S.C.T. a las patentes, una importancia reflejada tanto en su utilización como indicador de nuestro potencial innovador como en la preocupación que empiezan a sentir al respecto los propios centros públicos de investigación. Para las grandes empresas, y dependiendo del sector, las patentes no solo no son tan importantes (hasta el punto que su importancia puede derivar más bien del estímulo al «cliente interno»); sino que estas grandes empresas son conscientes de que pueden ser un obstáculo a la innovación en el sentido de resultar en tecnologías subóptimas que se adoptan como compromisos forzados por el uso oportunístico de los derechos de propiedad acumulados. A mi juicio corremos el peligro de satisfacernos con la convergencia en cifra de patentes o con la mejora en la balanza tecnológica cuando, tal como yo he insinuando, esa protección genera un monopolio temporal que no sólo

11 Véase The Magic Cauldron, un imaginativo trabajo de Eric S. Raymond que aparece en su famoso The Cathedral and the Bazaar. El trabajo puede descargarse de su página web. 
es malo para el consumidor; sino que además puede frenar la puesta en común de resultados que otros podrían explotar o frenar el propio esfuerzo investigador. Pero, dejando esto a un lado, paso ahora a relacionar estas ideas novedosas sobre propiedad intelectual con la R.S.C..

Pero para ello tengo que recordar unas ideas sobre señalización propias de la Economía de la Información. Una acción cualquiera funciona como una señal creíble solo cuando sería irracional tomarla a no ser que sea cierto lo que pretendo señalar (hacer un doctorado en una Universidad dura es una señal de inteligencia pues de lo contrario sería demasiado costoso como para sacarle rentabilidad). Pues bien pensemos ahora que estamos en un mundo como el real en el que funcionan las patentes y el copyright y preguntémonos si la renuncia a esos derechos puede constituir una señal propiamente dicha.

En el mundo cultural no se dan las condiciones de Eric Raymond expuestas más arriba y, en consecuencia, el copyright puede ser necesario si queremos contar con productos culturales. Por lo tanto renunciar a los derechos de autor (si lo permitiera la legislación) es una señal de la calidad del producto. En un mundo así cabe un equilibrio separador en el que los precios son tales que permiten distinguir los productos buenos de los malos. El que se sabe bueno renuncia al copyright, señalando así su calidad y permitiendo cargar un precio que varía con las condiciones de la demanda. Si esta es poco elástica le permite cargar un precio alto y ganar mucho por lo que renunciaría a sus derechos de autor. Si la demanda es muy elástica podría bajar el precio; pero en este caso todos le imitarían y la señal dejaría de funcionar. En todo caso si se da un equilibrio separador esto es bueno para el consumidor y para el innovador que mejoran su información y, finalmente para la productividad y la creación de riqueza.

En el mundo industrial se dan las condiciones de Raymond y como entonces la patente no es necesaria, renunciar a ella no es ninguna señal; el equilibrio ya no es separador; sino del tipo pooling (mezclado) en el que los precios no permiten distinguir la calidad del producto. Esto es malo para el consumidor y el innovador en términos de información y finalmente ralentiza la innovación y frena la mejora de la productividad y la creación de riqueza.

Pues bien, si mi análisis hasta aquí es correcto, la R.S.C. cobra un interés inusitado más allá de su aspecto de remedo del cuidado real de los stakeholders o de su uso como mecanismo de prestigio o fidelización. En efecto, el mecenazgo como parte de la R.S.C. ha ido dirigido fundamentalmente al mundo cultural, aquel que lo necesita menos puesto que en él hay señales que permiten subsistir a los buenos. En cambio en el mundo de la ciencia, tan necesario para la innovación industrial, la 
R.S.C. — con excepciones - se ha volcado menos a pesar de que, al no admitir señales, necesita más apoyo a los buenos. En mi opinión la gran empresa se ha limitado en este punto a subvencionar a algunos centros de investigación a efectos de generar profesionales y/o prescriptores sin que se haya hecho esta conexión de manera general. Por lo tanto se podría pensar que la gran empresa española continúa considerando la R.S.C. como una obligación social a veces impuesta por un dirigismo mal entendido y más o menos relacionada con el buen gobierno y, en cualquier caso, exagerada cuando el objetivo empresarial ya tiene connotaciones sociales. Sería bueno que hicieran esta conexión y que programas como el CENIT, del que nuestras grandes empresas no parecen esperar gran cosa, colaboren a que estas implicaciones novedosas se aclaren. El apoyo decidido del mecenazgo a la ciencia puede ir cambiando el origen de la financiación en la dirección adecuada, es coherente con las posibles privatizaciones de la generación de ciencia básica y colabora a unificar el lenguaje científico y el empresarial.

\section{Conclusiones}

La innovación es crucial en el mundo de hoy caracterizado por la globalización, la importancia del conocimiento y el uso extendido de las TIC. Además ha adquirido ya un status conceptual propio y un tratamiento sistemático que deriva en una atención específica a los S.C.T., una institución ésta en la que las empresas deberían jugar un papel importantísimo, especialmente las grandes. Las TIC han sido cruciales en la focalización del problema de la innovación a la que han facilitado de formas variadas. Por un lado aplanando la organización interna, lo que disminuye los costes de coordinación, un factor clave para la innovación. Por otro lado resulta que el mundo de hoy, global y cargado de conocimiento, puede fomentar la creatividad (crucial para la innovación) a través del incremento de la promiscuidad, intergeneracional, social y cultural.

Caben para terminar algunos avisos y algunas recomendaciones. En el capítulo de avisos creo justo subrayar que, desde el punto de vista administrativo, además de prestar atención a las condiciones del «procurement», merecería la pena no imitar demasiado lo que ha ocurrido en los S.C.T de otros países. Estos se desarrollaron en un momento de la evolución del sistema económico que ya ha pasado. Por eso mismo estos S.C.T., incluido el nuestro, pueden estar mal enfocados hoy. Esto ocurre especialmente en el campo de la propiedad industrial. El S.C.T. español se entera de las ventajas del monopolio temporal que otorgan las patentes justo cuando éstas se 
ponen en duda como aceleradoras de la innovación y cuando el análisis coste beneficio se muestra como muy negativo. En efecto, Romer ${ }^{12}$ ha calculado que en la industria discográfica la pérdida de bienestar generada por el exceso del precio sobre el coste marginal (exceso propio del monopolio de la patente) es comparable en magnitud al ingreso total de esa industria. Y cuando, por otro parte un trabajo como el de Urrutia et al. ${ }^{13}$ ha mostrado con datos que no hay relación perceptible entre patentes y beneficio, una constatación que empieza a ser aceptada y a generalizarse.

Pasando ahora al capítulo de recomendaciones, hay una genérica. No hay porqué confiar en las empresas grandes per se. Estas, a salvo excepciones muy puntuales, han desaprovechado las posibilidades de extraer el conocimiento tácito y de gestionarlo provechosamente. También han desaprovechado la posibilidad de contratar con los suministradores de manera que se incremente la innovación y tampoco han sabido explotar la información sistemática proporcionada por la clientela. Salvo excepciones una vez más.

Cabe pues que nos saltemos algunas etapas y que reiniciemos el diseño de nuestro S.C.T. evitando algunos de los errores cometidos por quienes han ido por delante de nosotros. Pero quizá la recomendación más inesperada tenga que ver con la R.S.C. y con una nueva concepción del mecenazgo. Concentrando éste en el mundo científico nos acercaríamos a la deseada proporción $(1 / 3,2 / 3)$ a favor de las empresas y trabajaríamos a favor de la homogenización del lenguaje.

Y termino diciendo que, como creo que está claro que la oferta de planes públicos no ha cambiado a las empresas, habría que cambiar de táctica y ayudar solo a aquellas empresas que se hayan ayudado a sí mismas. Que no se les den fondos para investigar; sino que se les otorguen recompensas por haber investigado.

\section{REFERENCIAS BIBLIOGRÁFICAS}

Aghion, Ph.; Blundell, R. W.; Grifith, R.; Howit, P. y Prantl, S. (2005), «The effects of Entry on Incumbent Innovator and Productivity», CEPR Discussion Paper núm. 5323.

Baumol, W. J. (1951), Economic Dynamics, Nueva York, Macmillan.

- (2002), The Free-Market Innovation Machine, Princeton University Press.

Besley, T. y Ghatack, M. J. (2001), «Government versus Private Ownership of PublicGoods», CEPR, núm. 2725

12 Véase el artículo de Romer (2002). 
Boldrin, M. y Levine, D. (2002), «The Case Against Intellectual Property», American Economic Review, vol. 92, Papers and Proceedings, págs. 209-212.

Boldrin, M. y UrRutia, J. (2003), «¿Gedeprensa? No gracias», en Urrutia (2003).

DAVID, P. (1998), «Clio and the Economic Organization of Science: Common Agency Contracting and the Emergence of Open Science Institutions», American Economic Review, Papers and proceedings, págs. 15-21.

Dysson, F. ( 1985), Infinite in all directions, Nueva York, Harper and Row.

Hart, O. (1976), Firms, Contracts and Financial Structure, Oxford University Press.

Guimón, J. (2007), «La IED intensiva en I+D: Definiciones, Causas y Consecuencias», Cap. 2 de la Tesis doctoral en preparación. Disponible a través del autor, del presente trabajo.

Lafuente, A. y Oro, L. (1992), El sistema Español de Ciencia y tecnología. Evolución y Perspectivas, Madrid, Los Libros de Fundesco.

RaYmond, E. S. (1997), The Magic Cauldron, en www.Catb.org/esr/writings/cathedral-bazaar/ magic-cauldron/

Romer, P. (1990), «Endogenous Technological Change», Journal of Political Economy, vol. 98, núm. 5, part 2: The Problem of Development: A Conference of the Institute for the Study of Free Enterprise Systems. Oct.

- (2002), «When should we use Intellectual Property rights?», American Economic Review, vol. 92, Papers and Proceedings, págs. 213-216.

Schumpeter, J. A. (1942), Capitalism, Socialism and Democracy, 2. a edición, Nueva York, Harper and Brothers.

Solow, R. (1956), «A Contribution to the Theory of Economic Growth», Quarterly Journal of Economics, 70.

Urrutia, J. (1993), «Sistemas de Ciencia y Tecnología», Telos.

- (2003), Economía en Porciones, Madrid, Prentice-Hall.

- (2005), «Objets trouvés», Expansión, 20 de agosto.

- (2005), «Promiscuidad», Expansión, 6 de septiembre.

Urrutia, R.; Saurabn, V. y SAim, S. S. (200?), «Research focusing on relationship between Innovation, Research \& Devlopement and Patents of Innovative Companies», Penn State University, unpublished. Documento disponible a través del autor del presente trabajo. 